intrapericardial diaphragmatic hernia, because adhesions in the pericardium can be freed more easily. ${ }^{3-6}$ In our case, an abdominal approach was used. The reduction of the hernia was performed without difficulties.

The organ most frequently involved in traumatic intrapericardial diaphragmatic hernia is the transverse colon, followed by the stomach and greater omentum. ${ }^{3}$ Most authors recommend the closing of the hernial defect with nonabsorbable sutures.

\section{CONCLUSIONS}

Traumatic intrapericardial diaphragmatic hernia alone can be fatal if it is not recognized and treated promptly. The observation of a diaphragmatic defect on cross-sectional imaging is essential to the diagnosis of traumatic intrapericardial diaphragmatic hernia. Once it is identified, immediate surgi- cal intervention is required to reduce the hernia and repair the defect.

\section{References}

1. Fleyfel M, Ferreira JF, Gonzalez De Linares H, et al. Cardiac tamponade after intrapericardial diaphragmatic hernia. Br J Anaesthesiol. 1994;73:249.

2. De Rooij PD, Haarman HJ. Herniation of the stomach into pericardial sac combined with cardiac luxation caused by blunt trauma: a case report. $J$ Trauma. 1993;34:453.

3. Reina A, Vidaña E, Soriano P, et al. Traumatic intrapericardial diaphragmatic hernia: case report and literature review. Injury. 2001;32:153-6.

4. Girzadas DV, Fligner DJ. Delayed traumatic intrapericardial diaphragmatic hernia associated with cardiac tamponade. Ann Emerg Med. 1991;20: 1246 .

5. Barker JA, Gavant ML, Hughes CB. Posttraumatic intrapericardial diaphragmatic hernia. Am J Roentgenol. 1997;169:315.

6. Kuzucu A, Isxik B, Baysal T, Soysal O, Ulutasx H. Traumatic intrapericardial diaphragmatic hernia. Radiography. 2007;13:169-71

7. Wenzel DJ, Hamilton JD. Cross-sectional CT of strangulating intrapericardial diaphragmatic hernia. AJR Am J Roentgenol. 2001;177:686-8.

\title{
Identification by transesophageal echocardiography of intramural hematoma and the site of aortic rupture
}

\author{
Kazumasa Orihashi, MD, Taijiro Sueda, MD, Kenji Okada, MD, and Katsuhiko Imai, MD, Hiroshima, Japan
}

Computed tomography (CT) is a noninvasive method of assessing the entire aorta and is essential for making a rapid diagnosis of acute aortic dissection with high sensitivity and specificity. ${ }^{1}$ On occasion, however, CT does not adequately inform a surgical decision, and additional information is needed. We report a case in which information obtained by transesophageal echocardiography (TEE) was critical in plotting a strategy.

\section{CLINICAL SUMMARY}

An 86-year-old female patient with sudden onset of flank pain was transferred to our hospital because of an extraperitoneal hematoma detected with regular CT. In the radiology department, her blood pressure dropped to $50 \mathrm{~mm} \mathrm{Hg}$. The CT examination, carried out with the blood pressure below $90 \mathrm{~mm} \mathrm{Hg}$, suggested left hemothorax; however, neither aortic dissection nor aneurysm could be recognized (Figure 1). The patient's blood pressure dropped to $40 \mathrm{~mm}$ $\mathrm{Hg}$, and she was immediately transferred to the operating

\footnotetext{
From the Department of Cardiovascular Surgery, Hiroshima University Hospital, Hiroshima, Japan.

Received for publication Nov 13, 2007; accepted for publication Nov 26, 2007.

Address for reprints: Kazumasa Orihashi, MD, Cardiovascular Surgery, Hiroshima University Hospital, Kasumi 1-2-3, Minami-ku, Hiroshima 734-8551, Japan (E-mail: orichan@hiroshima-u.ac.jp).

J Thorac Cardiovasc Surg 2008;136:1089-91

$0022-5223 / \$ 34.00$

Copyright (C) 2008 by The American Association for Thoracic Surgery doi:10.1016/j.jtcvs.2007.11.058
}

room with a diagnosis of hemothorax of unknown etiology and rupture site.

The TEE assessment revealed aortic dissection in the middle portion of the descending aorta, but with the proximal and distal segments intact (Figure 2, $A$ ). There was no aneurysm or thick atheroma. In the mediastinal hematoma, an echo-free area was present adjacent to the aorta (Figure 2, $B)$. The surrounding hematoma showed pulsatile motion. A color Doppler signal was not detected in either the false lumen or the echo-free area. TEE indicated localized type $\mathrm{B}$ dissection with mild thrombosis of the rupture site.

The left thorax was entered at the fifth intercostal space. Because the descending aorta was diffusely covered by hematoma, the extent of dissection could not be determined by inspection but instead was precisely located by TEEguided palpation. Under conditions of partial bypass and mild hypothermia, the aorta was crossclamped and incised. The false lumen was seen to be filled with fresh clot, and no intimal tear was found. The mediastinal hematoma at this level was not solid. A 5-cm segment of aorta was replaced with a vascular prosthesis. The patient's postoperative course was uneventful, without clamp injury or paraplegia, and she was discharged without complications.

\section{DISCUSSION}

Aortic dissection was not recognized with CT because the densities of aortic wall and blood were nearly identical. In 




FIGURE 1. Computed tomographic scans showing massive left hemothorax. Neither aneurysm nor dissection is recognizable in these images.

contrast, TEE clearly showed dissection of aortic wall because the false lumen and blood in the thorax were less echogenic than the aortic wall.

With CT assessment, extravasation of contrast medium is essential in identifying the presence of rupture and rupture site. Such a finding can be absent if the rupture site is covered with fresh clot. The absence of a flow signal in the echo-free area suggests that a clot may have prevented extravasation of blood. Although the presence of shock may have been responsible for negligible extravasation, hypotension is not uncommon in acute aortic dissection, occurring in a third of cases in our experience. The rupture site could be located with TEE as an echo-free area adjacent to the dissected aortic wall and surrounded by nonsolid hematoma with pulsatile motion. The information on tissue properties and motion provided by TEE was beneficial in this case.

TEE was also advantageous for minimizing the invasiveness of the surgical approach by clarifying the extent of dissection. If involvement of the arch had not been ruled out, open proximal anastomosis with deep hypothermic circulatory arrest might have been chosen. The extra information allowed the length of aortic replacement to be minimized in this critically ill, elderly patient. The surgeon could precisely recognize the extent of dissection by palpating the aorta under


FIGURE 2. Transesophageal echocardiograms showing short-axis view of descending aorta. A, Aortic dissection, hemothorax, and mediastinal hematoma are apparent. B, Echo-free area in mediastinal hematoma adjacent to aorta indicates rupture site. 
TEE guidance. TEE may also be beneficial for reducing the risk of paraplegia.

Although the priority of $\mathrm{CT}$ assessment in acute aortic dissection is not in dispute, TEE may be a useful adjunct to overcome its limitations.

\section{Reference}

1. Shiga T, Wajima Z, Apfel CC, Inoue T, Ohe Y. Diagnostic accuracy of transesophageal echocardiography, helical computed tomography, and magnetic resonance imaging for suspected thoracic aortic dissection: systematic review and meta-analysis. Arch Intern Med. 2006;166:1350-6.

\title{
Aortoesophageal fistula associated with a Kommerell diverticulum and right-sided aortic arch
}

\author{
Karl G. Reyes, MD, ${ }^{\mathrm{a}}$ Inderjit S. Gill, MD, ${ }^{\mathrm{a}}$ Thomas Temes, MD, ${ }^{\mathrm{a}}$ and Noreen C. Griffin, CNP, ${ }^{\mathrm{b}}$ Cleveland, Ohio
}

Aortoesophageal fistula (AEF) is rare and often fatal complication of vehicular trauma. In the past, it has also been reported in the presence of congenital arch anomalies. We report here a case of traumatic AEF in a patient with both a right-sided aortic arch and a Kommerell diverticulum, the first such in the literature, as well as its successful management by staged open surgical repair.

\section{CLINICAL SUMMARY}

A 26-year-old man was taken to the emergency department after a vehicular crash in which he had been the ejected, unrestrained driver. Injuries noted on admission were primarily intracranial, with a depressed mental status necessitating mechanical ventilation. The patient's mental status eventually improved to the point at which he could be weaned from the ventilator and required only a percutaneous endoscopic gastrostomy tube for feeding. He was discharged to a rehabilitation center 3 weeks later.

During the patient's stay at the rehabilitation center, approximately 4 weeks after the crash, he had massive gastrointestinal bleeding, with bright red blood coming out of his percutaneous endoscopic gastrostomy tube. The patient was taken to a nearby hospital, where he was resuscitated and subsequently taken to the endoscopy suite. On esophagogastroscopy, large amounts of blood and blood clots were seen, but with no identifiable source of bleeding. Because of the suddenness of the event, the diagnosis of AEF was considered, and the patient was flown to our institution. On arrival, the patient had no active bleeding. Diagnostic studies included chest radiography, results of which were unremark-

\footnotetext{
From the Department of Thoracic and Cardiovascular Surgery, The Cleveland Clinic Foundation, ${ }^{\mathrm{a}}$ and the Metrohealth Medical Center, ${ }^{\mathrm{b}}$ Cleveland, Ohio. Received for publication Nov 15, 2007; accepted for publication Nov 25, 2007.

Address for reprints: Karl G. Reyes, MD, Department of Thoracic and Cardiovascular Surgery, The Cleveland Clinic Foundation, 9500 Euclid Ave H35, Cleveland, OH 44195 (E-mail: reyesk2@ccf.org).

J Thorac Cardiovasc Surg 2008;136:1091-3

$0022-5223 / \$ 34.00$

Copyright $(C) 2008$ by The American Association for Thoracic Surgery doi:10.1016/j.jtcvs.2007.11.057
}

able except for findings suggestive of a right-sided aortic arch. Chest CT scan (Figure 1) confirmed the presence of a right-sided aortic arch and descent of the aorta on the right side of the chest. In addition, there was an aberrant left subclavian artery with an aneurysmal origin, consistent with a Kommerell diverticulum, and an anteriorly displaced esophagus that narrowed at the level of the aberrant left subclavian artery. The suspected AEF, however, was not seen on the CT scan. Esophagogastroscopy was repeated and this time showed a nonbleeding, pulsating, $0.7-\mathrm{cm}$ ulcer within the esophagus at $25 \mathrm{~cm}$, corresponding to the level of the aortic knuckle. The patient was then taken to the operating room.

En route to the operating room, the patient again had massive bleeding. A standard left posterolateral thoracotomy through the fourth interspace was done. The posterior mediastinum was exposed, and the preexisting congenital anomalies were identified. The esophagus was then mobilized until an area of adherence between the esophagus and aorta was found, at the level of the distal aortic arch and very close to the Kommerell diverticulum. Pledgeted sutures were then passed and tied down in this area. Intraoperative esophagoscopy confirmed the cessation of bleeding. At this point, although hemostasis had been achieved, the patient had hypothermia and severe acidosis. It was therefore decided that the esophageal injury would be managed later, and the patient was taken to the intensive care unit. The patient's postoperative course went remarkably well, and he was returned to the operating room on the 6th postoperative day. Before reopening of the thoracotomy, esophagogastroscopy was performed at the operating room, and the previously identified lesion was once again seen, as was pledget material that appeared to be present within the lesion. The left posterolateral thoracotomy was subsequently reopened. The esophagus was mobilized, and the fistula was excised with a small disk of esophagus left attached. The esophageal defect was then repaired primarily, and a nasogastric tube was inserted in position adjacent to the repair. On the aortic side, the polytetrafluoroethylene pledgets were removed and 